\title{
CONTINUOUS AND INTERMITTENT EXERCISE TRAINING RESPONSES IN LIVER AND WHITE ADIPOSE TISSUE AQUAGLYCEROPORINS
}

original paper

() Wroclaw University of Health and Sport Sciences

DOI: https://doi.org/10.5114/hm.2022.107310

\section{YADOLLAH NOORISHORABI ${ }^{1}$, ELAHE TALEBI-GARAKANI ${ }^{1}$, ALIREZA SAFARZADE ${ }^{1}$, SÍLVIA ROCHA-RODRIGUES ${ }^{2,3,4}$, SARKAWT KOLAHDOUZI ${ }^{1}$}

${ }^{1}$ Department of Exercise Physiology, Faculty of Physical Education and Sports Sciences, University of Mazandaran, Babolsar, Iran

${ }^{2}$ Escola Superior de Desporto e Lazer, Instituto Politécnico de Viana do Castelo, Viana do Castelo, Portugal

${ }^{3}$ Research Centre in Sports Sciences, Health Sciences and Human Development, Vila Real, Portugal

${ }^{4}$ Tumour and Microenvironment Interactions Group, Institute of Biomedical Engineering, i3S - Instituto de Investigação e Inovação em Saúde, University of Porto, Porto, Portugal

\section{ABSTRACT}

Purpose. We analysed the effects of moderate-intensity continuous training (MICT) and high-intensity interval training (HIIT) on hepatic and adipose tissue aquaglyceroporins (AQPs) in rats fed with high-fat diet (HFD).

Methods. Overall, 48 male Wistar rats were fed with a normal diet (ND, 10.4 kcal\% fat) or HFD (62.1 kcal\% fat) over 10 weeks. Then, the animals were divided into 6 groups: ND sedentary (NS), N + MICT, N + HIIT, HS, H + MICT, and H + HIIT. The trained animals performed 10-week matched distances of MICT and HIIT on a motorized treadmill (5 times/week) while maintaining dietary treatments. The liver and epididymal white adipose tissue (eWAT) were investigated to determine triglycerides (TG) and AQP7 and AQP9 levels.

Results. HFD increased body weight, liver and eWAT weight, plasma insulin and glucose levels, and insulin resistance. Both MICT and HIIT were able to decrease body weight and liver and eWAT weight in the HFD-fed group. HFD increased plasma TG, glucose, and insulin levels, attenuated by MICT and HIIT programs. HFD increased TG content and AQP7 and did not alter AQP9. MICT and HIIT programs decreased hepatic TG content and AQP7 and AQP9 levels in ND-fed animals. In HFD-fed animals, only MICT decreased AQP9, and both MICT and HIIT decreased TG content and AQP7 levels in eWAT. Conclusions. Our findings suggest that the regulation of adipose tissue AQP7 and hepatic AQP9 by both MICT and HIIT interventions can have a significant effect on fat metabolism and glucose homeostasis.

Key words: exercise, HIIT, obesity, aquaglyceroporins, hepatic tissue

\section{Introduction}

In white adipocytes, lipogenesis and lipolysis occur as a response to whole body energy balance. Under excessive calories conditions, the adipose tissue stores triglycerides (TG), while in fasting and/or sympathetic nerve-activation states, it provides free fatty acids and glycerol to the energy expenditure organs [1, 2]. Nevertheless, in a situation of an excess of calories and low levels of physical exercise, an increased fat accumulation can occur [3], which can lead to the development of obesity and associated disorders, such as insulin resistance and fatty liver disease [2, 3]. Adipocyte lipoly- sis can occur intracellularly after glycerol crosses the plasma membrane, which requires specific proteins called aquaglyceroporins (AQPs), a subfamily of the water channel proteins aquaporins [1]. Therefore, AQPs expression and functioning play a key role in the fat accumulation in the associated metabolic alterations $[1,2]$. In type 2 diabetic patients, the expression of visceral adipose tissue AQP7 was elevated and correlated with increased lipolysis and adipocyte hypertrophy [4]. The relevance of AQP9 in glucose and glycerol metabolism and its regulation by insulin has been previously investigated by Kuriyama et al. [5]. These authors demonstrated an inverse relationship between

Correspondence address: Sílvia Rocha-Rodrigues, Complexo Desportivo e de Lazer Monte Prado, Escola Superior de Desporto e Lazer, Instituto Politécnico de Viana do Castelo, Rua Escola Industrial e Comercial de Nun'Alvares, 4900-347, Viana do Castelo, Portugal, e-mail: silviadarocharodrigues@gmail.com

Received: January 28, 2020

Accepted for publication: May 17, 2021

Citation: Noorishorabi Y, Talebi-Garakani E, Safarzade A, Rocha-Rodrigues S, Kolahdouzi S. Continuous and intermittent exercise training responses in liver and white adipose tissue aquaglyceroporins. Hum Mov. 2022;23(1):105-112; doi: https:// doi.org/10.5114/hm.2022.107310. 


\section{HUMAN MOVEMENT}

Y. Noorishorabi et al., Effects of HIIT on aquaglyceroporins

circulating insulin and hepatic AQP9 levels, but in the liver of diabetic mice AQP9 is over-expressed. On the other hand, the reduction of hepatocyte AQP9 and, consequently, glycerol permeability might be a defensive mechanism to counteract further fat infiltration in liver parenchyma in obese leptin-deficient $(o b / o b)$ mice, a model of non-alcoholic fatty liver disease.

Exercise training (ET) has been recognized as an important strategy for preventing obesity and improving insulin resistance along with profound effects on white adipose tissue (WAT) and liver homoeostasis [6]. In response to ET, the glycerol from lipolysis represents an important substrate for gluconeogenesis and thus the idea of ET influencing AQPs in WAT and liver is promising. However, few studies have investigated the hypothetical modulation of AQPs in response to physical exercise. In rats with diet-induced obesity, RochaRodrigues et al. [7] reported that 8 weeks of ET decreased glycerol levels and epididymal WAT (eWAT) AQP7, while 17 weeks of voluntary wheel running did not, suggesting that modulation of AQP7 in response to ET may be intensity-dependent. In individuals without predisposition to type 2 diabetes, 10 -week ET at $70 \%$ maximal oxygen uptake $\left(\mathrm{VO}_{2} \max \right)$ was accompanied by a 2.2-fold increase in the subcutaneous adipose tissue AQP7 content in women and a 48\% decrease in men [8]. In non-diabetic obese female patients, ET had no impact on AQP7 or AQP9 mRNA expression in subcutaneous adipose tissue [9]. The impact of distinct models of ET on AQP7 and AQP9 as a possible therapeutic target for preventing and treating obesity and associated metabolic disorders is unclear.
Therefore, the purpose of this study was to evaluate the effect of moderate-intensity continuous training (MICT) and high-intensity interval training (HIIT) on hepatic tissue AQP9 and adipose tissue AQP7 in animals with obesity induced by high-fat diet (HFD).

\section{Material and methods}

\section{Animals, diets}

A total of 48 male Wistar rats (age: 4-6 weeks, body weight: $134.64 \pm 28.47 \mathrm{~g}$ ) were purchased. The rats were allocated in standard cages ( 4 rats per cage) and maintained in a pathogen-free room (12-hour light/ dark cycle), in constant temperature $\left(24-26^{\circ} \mathrm{C}\right)$ and humidity (55 $\pm 4 \%$ ), with unrestricted access to water and food.

After 1-week adaptation to the laboratory environment, the animals were fed ad libitum with a nutritionally normal diet (ND, $10.4 \mathrm{kcal} \%$ fat) or HFD (62.1 kcal\% fat), as described elsewhere [10], over a 10-week period. Thereafter, the rats were divided into 6 groups ( $n=8 /$ group): normal diet sedentary (NS), normal diet MICT $(\mathrm{N}+\mathrm{MICT})$, normal diet HIIT $(\mathrm{N}+$ HIIT), HFD sedentary (HS), HFD MICT (H + MICT), and HFD HIIT (H + HIIT) (Figure 1).

\section{Exercise training protocols}

In order to understand the therapeutic effects of both MICT and HIIT, we applied the ET programs in the animals after 10 weeks of dietary treatment since

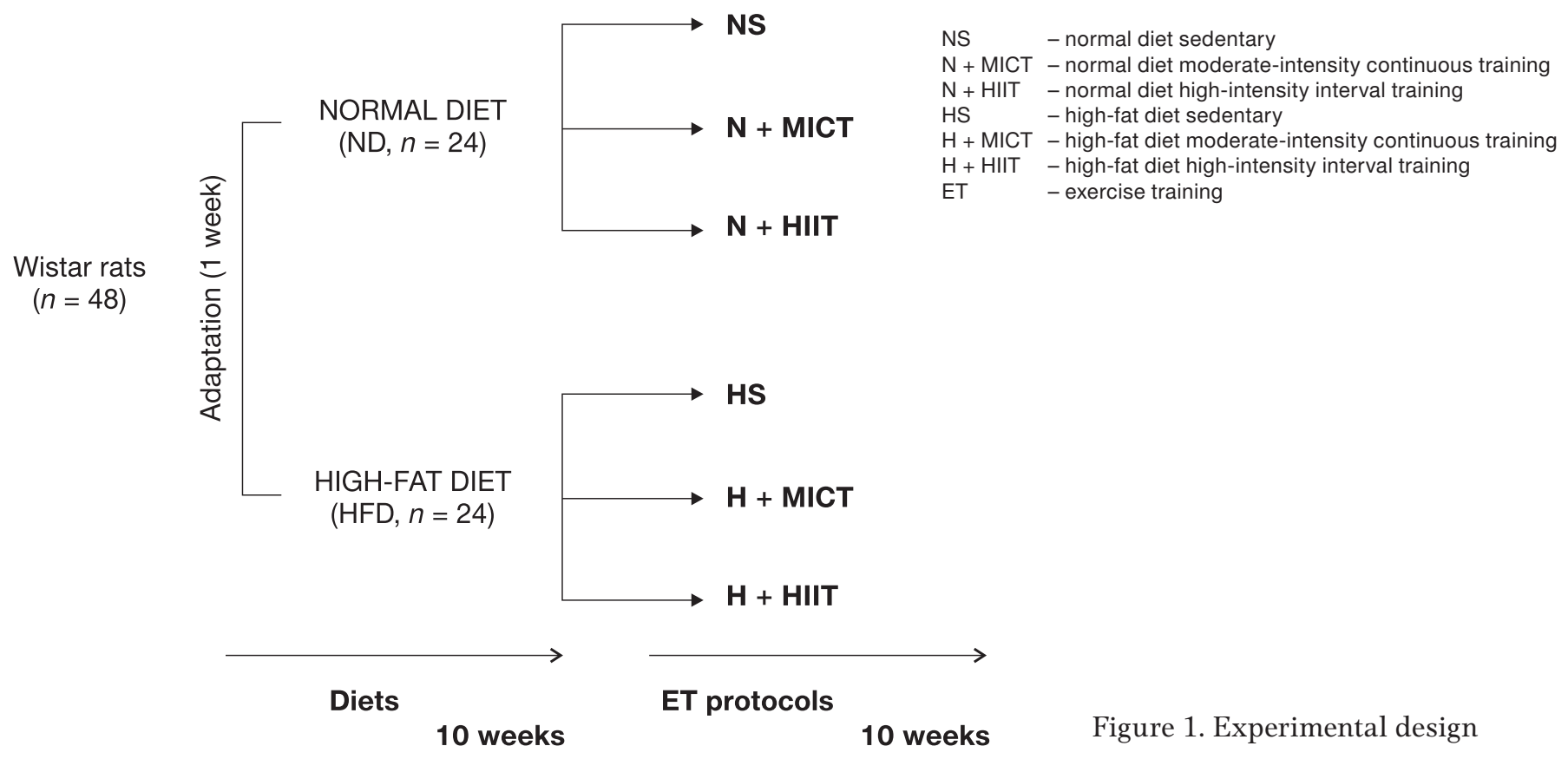


at this time point, HFD-fed rats already exhibited obesity-related metabolic features, in accordance with previous findings $[6,10]$. The ET programs were performed on a motorized treadmill, 5 days per week, for 10 weeks. The MICT groups were progressively increased from $5 \mathrm{~min}$ per day at $15 \mathrm{~m} \cdot \mathrm{min}^{-1}$ with a $15 \%$ inclination up to $60 \mathrm{~min}$ per day at $20 \mathrm{~m} \cdot \mathrm{min}^{-1}$ with a $15 \%$ inclination in the last 5 weeks of the program. In the HIIT groups, the animals ran 6 bouts of 4 min at $40 \mathrm{~m} \cdot \mathrm{min}^{-1}$ with a $15 \%$ inclination and 3 min of rest. The protocols were originally designed to have the same total running distance for all groups, as proposed by Maillard et al. [11].

\section{Blood and tissue collections}

The animals were fasted overnight for 12 hours, with free access to drinking water. To avoid acute effects of ET, the exercised rats were sacrificed 48 hours after the last training session. After anaesthesia, blood samples were collected from the heart, centrifuged to separate plasma (1500 g for $10 \mathrm{~min}$ at $\left.4^{\circ} \mathrm{C}\right)$, and stored at $-20^{\circ} \mathrm{C}$ for biochemical analyses. Liver and visceral WAT - eWAT deposition - were quickly excised and stored at $-80^{\circ} \mathrm{C}$ until analysed.

Assessment of insulin resistance and lipid profile

Plasma glucose was determined with an enzymatic (GOD-PAP, glucose oxidase - aminoantipyrine) colorimetric method. Plasma insulin level was measured by using a commercial ELISA kit (Mercodia AB, Uppsala, Sweden, CT\#10-1250-01). Insulin resistance during fasting state was determined with a homeostasis model assessment of insulin resistance (HOMA-IR) calculated with the following formula: fasting insulin $\left(\mu \mathrm{U} \cdot \mathrm{ml}^{-1}\right) \times$ fasting glucose $\left(\mathrm{mmol} \cdot \mathrm{l}^{-1}\right) / 22.5$ [12]. The enzymatic colorimetric method with commercial kits was applied to measure TG content in plasma, liver, and in eWAT. The coefficient of variation and sensitivity of the measurement method were $2.4 \%$ and $1 \mathrm{mg}$ in $100 \mathrm{ml}$ for TG, and $2.3 \%$ and $5 \mathrm{mg} / \mathrm{dl}$ for glucose.

\section{Aquaglyceroporins determination}

Tissue samples were homogenized in 5 volumes of buffer containing $0.9 \% \mathrm{NaCl}, 50 \mathrm{mM}$ Tris- $\mathrm{HCl}$, and $12 \mathrm{M}$ leupeptin by using a Potter-Elvehjem homogenizer set at $1500 \mathrm{rpm}$ and cooled in ice. The homogenates were centrifuged at $9000 \mathrm{rpm}$ for $10 \mathrm{~min}$ at $4^{\circ} \mathrm{C}$.
Supernatants were collected and used for AQP analysis. ELISA kits specific for the rat were used to determine AQP7 (My Bio Source, USA, MBS728355) and AQP9 (My Bio Source, USA, MBS9344422) in liver and in eWAT, respectively, in accordance with the manufacturer's instructions.

\section{Statistical analysis}

Data are expressed as mean \pm standard error of mean. The effect of diet and ET interventions was investigated with 2-way analysis of variance (ANOVA). The Bonferroni post-hoc test was applied for post-hoc comparisons between groups and differences were considered significant at $p<0.05$. The statistical analyses were performed with the SPSS 24.0 for Windows software (SPSS Inc.).

\section{Ethical approval}

The research related to animal use was performed in accordance with the guidelines of the Institutional Animal Care and Use Committee and of the Federation of European Laboratory Animal Science Associations.

\section{Results}

The initial body weight of the animals before the beginning of diet and ET protocols was $134.64 \pm$ $28.47 \mathrm{~g}$. After 10 weeks of HFD, the final body weight and liver and eWAT weights increased in the HS group compared with the NS group. Both MICT and HIIT programs significantly decreased eWAT weight in the ND-fed group (N + MICT vs. NS; N + HIIT vs. NS), as well as decreased body weight and liver and eWAT weights in the HFD-fed group (H + MICT vs. HS; H + HIIT vs. HS), as depicted in Table 1.

Under normal diet conditions, both MICT and HIIT decreased plasma TG and insulin levels ( $\mathrm{N}+$ MICT and $\mathrm{N}+$ HIIT vs. NS). HFD increased plasma TG, glucose, and insulin levels, which were attenuated by both MICT and HIIT programs (H + MICT and $\mathrm{H}+$ HIIT vs. HS). HOMA-IR is a valid and well-established reproducible method for the assessment of insulin resistance in obese animals [12]. In the present study, HFD increased HOMA-IR, which was decreased by both ET interventions compared with the sedentary setting (Figure 2).

To gain further insight into the molecular mechanisms involved in the lipid accumulation regulation induced by physical exercise, we next analysed the levels of AQP7 and AQP9, two specific glycerol transporters in adipocytes and hepatocytes, respectively 


\section{HUMAN MOVEMENT}

Y. Noorishorabi et al., Effects of HIIT on aquaglyceroporins

Table 1 . The weights of body, liver, and epididymal white adipose tissue

\begin{tabular}{|c|c|c|c|c|c|c|c|c|c|}
\hline \multirow{2}{*}{ Parameters } & \multirow{2}{*}{ NS } & \multirow{2}{*}{$\mathrm{N}+\mathrm{MICT}$} & \multirow{2}{*}{$\mathrm{N}+\mathrm{HIIT}$} & \multirow{2}{*}{ HS } & \multirow{2}{*}{$\mathrm{H}+\mathrm{MICT}$} & \multirow{2}{*}{$\mathrm{H}+\mathrm{HIIT}$} & \multicolumn{3}{|c|}{$p$} \\
\hline & & & & & & & $\mathrm{D}$ & ET & $\mathrm{D} \times \mathrm{ET}$ \\
\hline $\begin{array}{r}\text { Initial body } \\
\text { weight (g) }\end{array}$ & $138.42 \pm 11.26$ & $135.42 \pm 11.08$ & $133.42 \pm 29.23$ & $136.28 \pm 13.07$ & $136.71 \pm 10.38$ & $132.57 \pm 11.07$ & 0.907 & 0.816 & 60.963 \\
\hline $\begin{array}{l}\text { Final body } \\
\text { weight }(\mathrm{g})\end{array}$ & $415.14 \pm 13.81$ & $374.71 \pm 11.04$ & $388.28 \pm 9.59$ & $498.85 \pm 13.76^{a}$ & $416.85 \pm 8.67^{\mathrm{b}}$ & $415.85 \pm 12.22^{b}$ & $<0.001$ & $<0.001$ & 10.057 \\
\hline $\begin{array}{l}\text { Liver } \\
\text { weight (g) }\end{array}$ & $12.52 \pm 0.40$ & $10.46 \pm 0.29$ & $11.07 \pm 0.41$ & $15.64 \pm 0.33^{\mathrm{a}}$ & $11.02 \pm 0.23^{\mathrm{b}}$ & $13.56 \pm 0.98^{\mathrm{b}}$ & $<0.001$ & $<0.001$ & 10.799 \\
\hline $\begin{array}{l}\text { eWAT } \\
\text { weight (g) }\end{array}$ & $8.59 \pm 1.06$ & $4.17 \pm 0.27^{\mathrm{a}}$ & $4.45 \pm 0.40^{\mathrm{a}}$ & $13.76 \pm 1.79^{a}$ & $7.01 \pm 0.45^{\mathrm{b}}$ & $8.28 \pm 0.74^{b}$ & $<0.001$ & $<0.001$ & 10.473 \\
\hline
\end{tabular}

Data are expressed as mean \pm standard error of mean.

eWAT - epididymal white adipose tissue, NS - normal diet sedentary

$\mathrm{N}+\mathrm{MICT}$ - normal diet moderate-intensity continuous training

$\mathrm{N}+\mathrm{HIIT}$ - normal diet high-intensity interval training

D - diet,

HS $\quad-$ high-fat diet sedentary

ET - exercise training

$\mathrm{H}+\mathrm{MICT}$ - high-fat diet moderate-intensity continuous training

$\mathrm{H}+\mathrm{HIIT}$ - high-fat diet high-intensity interval training

Significance at $p<0.05 ;{ }^{\text {a }}$ vs. NS, ${ }^{\text {b }}$ vs. HS
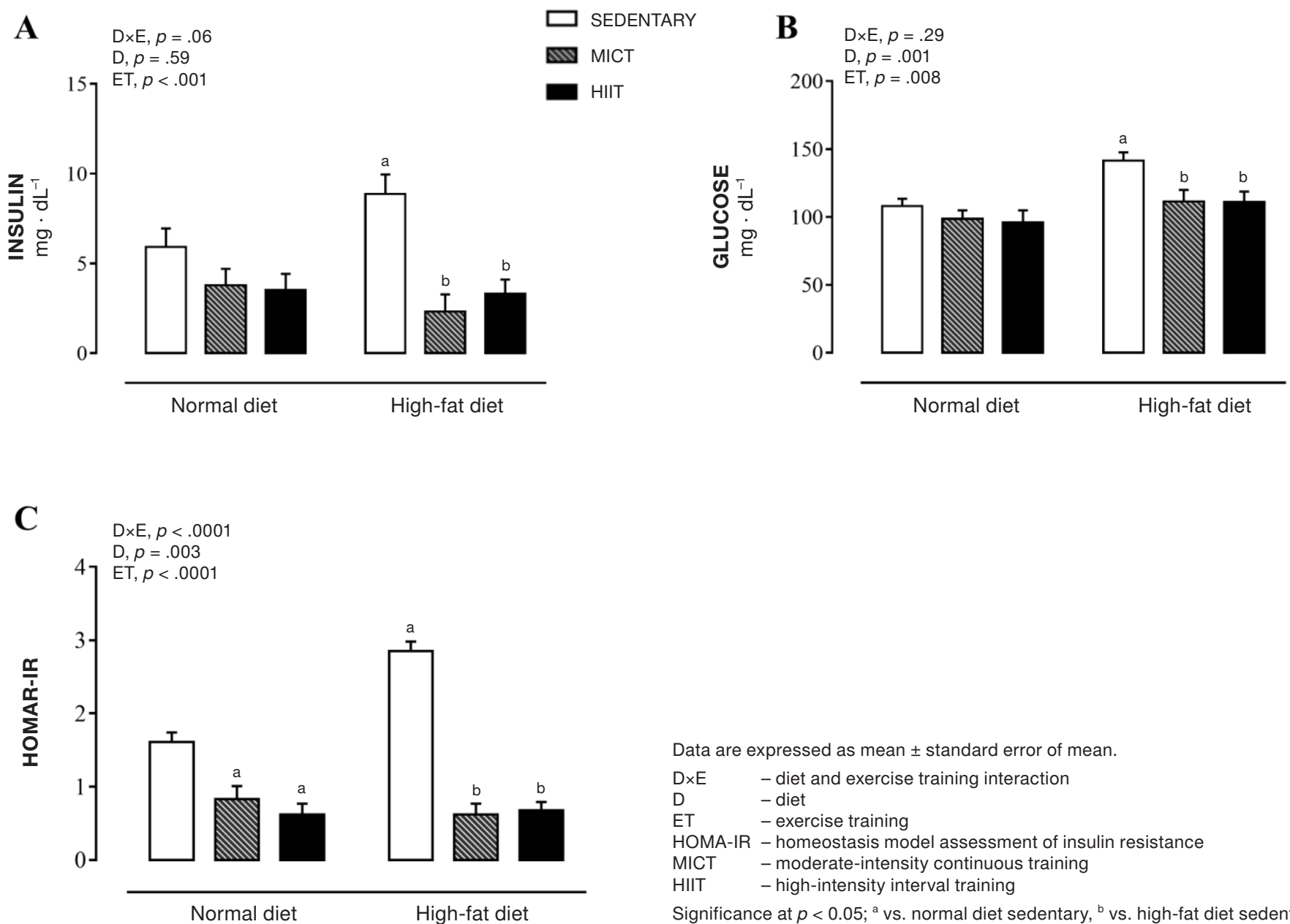

Figure 2. Plasma insulin (A) and glucose (B) levels, as well as HOMA-IR (C) 
$\mathbf{A}$

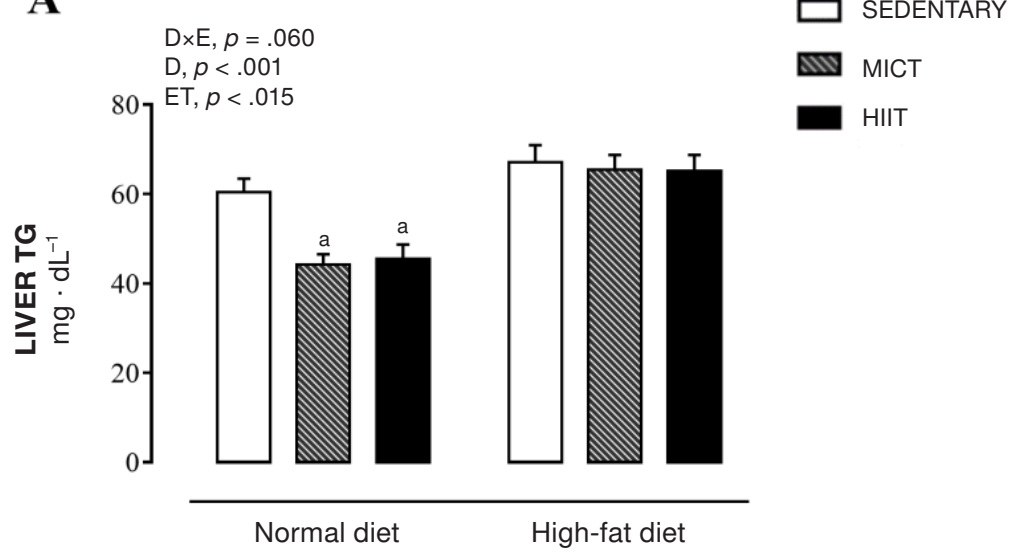

C

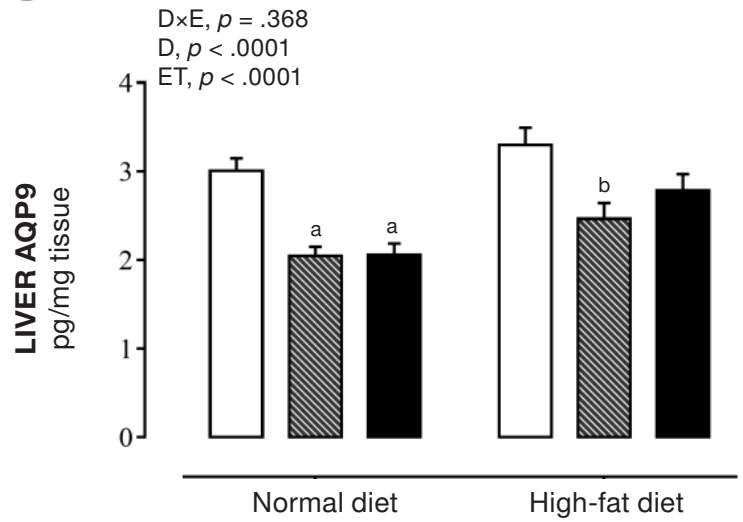

B

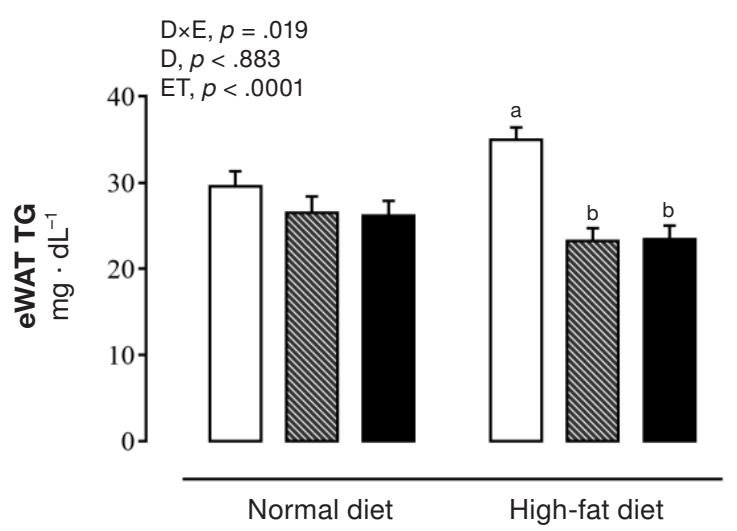

D

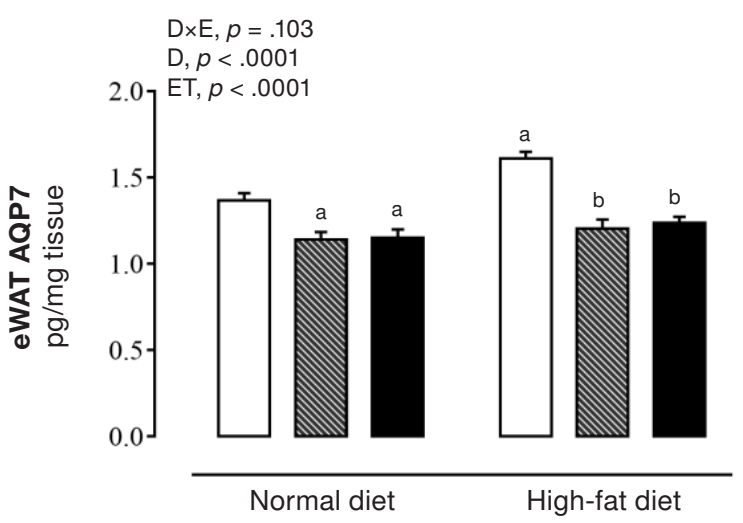

Data are expressed as mean \pm standard error of mean.

DxE - diet and exercise training interaction eWAT - epididymal white adipose tissue

$\mathrm{D}$ - diet

ET - exercise training

TG - triglycerides HIIT - high-intensity interval training
Significance at $p<0.05 ;{ }^{\text {a }}$ vs. normal diet sedentary, ${ }^{\text {b }}$ vs. high-fat diet sedentary

AQP - aquaglyceroporin

Figure 3. The content of TG (A, B), AQP9 (C), and AQP7 (D) in liver and eWAT

$[1,13]$. TG content and AQP7 in eWAT, as well as AQP9 in the hepatic tissue remained unchanged in HS animals compared with the NS group. Both MICT and HIIT programs decreased liver TG content and AQP7 and AQP9 levels in the N + MCIT and N + HIIT groups compared with the NS group. In HFD-fed animals, only MICT decreased AQP9 levels in the hepatic tissue, and both MICT and HIIT were able to decrease TG content and AQP7 levels in eWAT (Figure 3).

\section{Discussion}

The main findings of the present study suggest that animals submitted to 10-week HFD exhibited an increase of body weight and hepatic and WAT tissues concomitantly with metabolic alterations which strongly resemble the pathological features observed under the obesity context. On the other hand, both
MICT and HIIT reverted or, at least in part, attenuated some of the metabolic impairments imposed by HFDinduced obesity, such as decreased insulin resistance and reduced eWAT TG and AQP7 along with lower levels of TG in plasma.

Glycerol is synthesized from glucose via the glycolytic pathway $[1,13]$. In high glucose availability, which typically occurs in obesity, there is a steady supply of glycerol-3-phosphate for the synthesis of acylglycerols, produced by the lipogenic pathway, from glucose [14] or other substrates [1]. In most tissues, including WAT, acyl-coenzyme A can be alternatively synthesized from extracellular fatty acids, such as those released by lipoprotein lipase, which suggests that the production and release of glycerol from WAT play a key role in the regulation of fat metabolism, glucose homeostasis, and the associated metabolic disorders [15]. In this line, we observed that animals under the 
HFD regimen exhibited increased glucose levels and insulin resistance status, as well as increased TG in eWAT. A study by Kishida et al. [16] provided the first evidence for the function of AQP7 in the release of glycerol from adipocytes, which is regulated by several hormones, including insulin or catecholamines [1]. The gene expression of AQP7 and AQP9 in both white adipose and hepatic tissue has been demonstrated to be inversely regulated by plasma insulin levels [15]. Although the data concerning AQP7 levels obtained in the present study are not in agreement with previous research [5], it has been reported that WAT AQP7 and liver AQP9 expression is more abundant in obese mice and in Otsuka Long-Evans Tokushima Fatty rats with insulin resistance in spite of hyperinsulinemia [17, 18], which is in line with the findings of the present study. These animals display increased glycerol release from WAT in parallel with the increase of Aqp 7 mRNA [5], and also increased glycerol levels in the portal vein [19]. The high glycerol levels in the portal vein cause gluconeogenesis and result in hyperglycaemia through the pathological induction of liver AQP9 [5]. Thus, an increased AQP9 level in the liver of HFD-fed animals would be expected in the present study. However, WAT is highly sensitive to lipolysis induced by catecholamines [20] and owing to the stimulation of these hormones, the lipolysis of this tissue accelerates and significantly increases the permeability of fatty acids and glycerol to the bloodstream [21, 22]; this may be explained in part by the absence of alterations in the expression of AQP9 in the hepatic tissue. In fact, some studies demonstrated a decreased AQP9 content and hepatic glycerol permeability in obesity and insulin resistance [23, 24]. Cai et al. [25] showed that the knockdown of hepatic AQP9 alleviated HFD-induced nonalcoholic fatty liver disease in rats, suggesting a potential role of this protein in the pathogenesis of hepatic steatosis. Therefore, further studies are required to comprehend the molecular mechanisms involved in physiological and pathological coordinated regulation of organ-specific AQP7 and AQP9 in the prevention and/or treatment of obesity and associated disorders.

Few studies have investigated the hypothetical modulation of AQPs in response to ET. We were first to demonstrate that 2 types of ET, namely MICT and HIIT, markedly impacted on AQPs levels in liver and WAT under normal diet and HFD regimens. Similar to our findings, Rocha-Rodrigues et al. [7] reported decreased AQP7 mRNA and protein in eWAT after 8 weeks of ET [26]. ET intensity, in some degree, seems to have an important influence on AQP7 suppression by stimulating catecholamines, as demonstrated by Londos et al. [27]. In a study by Lebeck et al. [8], 10 weeks of ET, 45 min at $70 \%$ of $\mathrm{VO}_{2}$ max, decreased by $48 \%$ AQP7 protein content in subcutaneous WAT of individuals without predisposition to diabetes mellitus type 2. In turn, Trachta et al. [9] reported no changes in Aqp7 or Aqp9 genes after 3 months of a supervised exercise program of $30 \mathrm{~min}$ of ET ( 3 times per week) in non-diabetic obese female patients. Moreover, in the present study, the reduction of AQP7 and AQP9 levels was accompanied by a decrease of TG in eWAT of HFD-fed animals and in the liver only in ND-fed rats, which corroborates other studies [7]. These data suggest that under HFD conditions, the effects of MICT and HIIT were more pronounced in WAT than in the liver, which confirms that ET protocols should be longer after inducing obesity in order to be effective in the latter tissue. Generally, MICT and HIIT have a similar effectiveness in counteracting the HFD-induced metabolic disturbances, as previously demonstrated by Wewege et al. [28].

Collectively, the data suggest that exercise intensity and duration are important factors in inducing AQP alterations in both hepatic and WAT tissue and may constitute a potential mechanism allowing a better understanding of fat accumulation, glucose homeostasis, and other important functions in the obesity context. Moreover, only MICT decreased AQP9 levels in the hepatic tissue, but not TG levels in HFD-fed animals. This finding may be related with the TG synthesis in the liver likely due to de novo lipogenesis, glucose converted to malonyl-CoA, which only accounts for $26 \%$ of hepatic TG accumulation, whereas $60 \%$ is derived from plasma non-esterified fatty acids [29]. It is well documented that glycerol, which is needed to TG synthesis, can be derived from glycolysis pathway. So, changes in AQP9 cannot warrant liver TG reduction. Further studies are required to address this issue and help to better understand the obesity-related pathways in lipid metabolism.

In summary, our findings suggest that the regulation of adipose tissue AQP7 and hepatic AQP9 by both MICT and HIIT can have a significant effect on fat metabolism and glucose homeostasis. Thus, regulating AQP7 and AQP9 through ET can be considered as an interesting therapeutic strategy for controlling obesity and associated metabolic disorders.

\section{Conclusions}

Considering the relevant role of AQPs in fat metabolism, we contribute with novel data showing that HFD feeding induced alterations in AQP content in 
eWAT, suggesting an impairment on fat accumulation. This information increases knowledge of obesity-related mechanisms, helping understand the hypothetic role of both MICT and HIIT in hepatic and adipose tissue AQPs of HFD-fed rats. We show for the first time that both MICT and HIIT reverted or, at least in part, attenuated some of the metabolic impairments imposed by HFD-induced obesity, such as decreased insulin resistance and reduced eWAT TG and AQP7 along with lower levels of TG in plasma. With these findings, we contribute to better understanding of how physical exercise induces favourable effects on relevant metabolically active tissues, eWAT and liver, in the obesity context.

\section{Funding}

This work is supported by national funding through the Portuguese Foundation for Science and Technology, I.P., under project UID/DTP/04045/2019, to the Research Centre in Sports Sciences, Health Sciences and Human Development.

\section{Disclosure statement}

No author has any financial interest or received any financial benefit from this research.

\section{Conflict of interest}

The authors state no conflict of interest.

\section{References}

1. Rodríguez A, Catalán V, Gómez-Ambrosi J, Frühbeck G. Aquaglyceroporins serve as metabolic gateways in adiposity and insulin resistance control. Cell Cycle. 2011;10(10):1548-1556; doi: 10.4161/cc.10.10.15672.

2. Costa R, Rodrigues I, Guardão L, Rocha-Rodrigues S, Silva C, Magalhães J, et al. Xanthohumol and 8-prenylnaringenin ameliorate diabetic-related metabolic dysfunctions in mice. J Nutr Biochem. 2017;45:3947; doi: 10.1016/j.jnutbio.2017.03.006.

3. Frühbeck G, Méndez-Giménez L, Fernández-Formoso J-A, Fernández S, Rodríguez A. Regulation of adipocyte lipolysis. Nutr Res Rev. 2014;27(1):63-93; doi: 10.1017/S095442241400002X.

4. Rodríguez A, Catalán V, Gómez-Ambrosi J, GarcíaNavarro S, Rotellar F, Valentí V, et al. Insulin- and leptin-mediated control of aquaglyceroporins in human adipocytes and hepatocytes is mediated via the PI3K/ Akt/mTOR signaling cascade. J Clin Endocrinol Metab. 2011;96(4):E586-E597; doi: 10.1210/jc.2010-1408.

5. Kuriyama H, Shimomura I, Kishida K, Kondo H, Furuyama $\mathrm{N}$, Nishizawa $\mathrm{H}$, et al. Coordinated regulation of fat-specific and liver-specific glycerol channels, aquaporin adipose and aquaporin 9. Diabetes. 2002;51(10): 2915-2921; doi: 10.2337/diabetes.51.10.2915.
6. Gonçalves IO, Passos E, Rocha-Rodrigues S, Torrella JR, Rizo D, Santos-Alves E, et al. Physical exercise antagonizes clinical and anatomical features characterizing Lieber-DeCarli diet-induced obesity and related metabolic disorders. Clin Nutr. 2015;34(2):241247; doi: 10.1016/j.clnu.2014.03.010.

7. Rocha-Rodrigues S, Rodríguez A, Becerril S, Ramírez B, Gonçalves IO, Beleza J, et al. Physical exercise remodels visceral adipose tissue and mitochondrial lipid metabolism in rats fed a high-fat diet. Clin Exp Pharmacol Physiol. 2017;44(3):386-394; doi: 10.1111/14401681.12706.

8. Lebeck J, Østergård T, Rojek A, Füchtbauer E-M, Lund S, Nielsen S, et al. Gender-specific effect of physical training on AQP7 protein expression in human adipose tissue. Acta Diabetol. 2012;49(Suppl. 1):S215S226; doi: 10.1007/s00592-012-0430-1.

9. Trachta P, Drápalová J, Kaválková P, Toušková V, Cinkajzlová A, Lacinová Z, et al. Three months of regular aerobic exercise in patients with obesity improve systemic subclinical inflammation without major influence on blood pressure and endocrine production of subcutaneous fat. Physiol Res. 2014;63(Suppl. 2):299_ 308; doi: 10.33549/physiolres.932792.

10. Ahmadi-Kani Golzar F, Fathi R, Mahjoub S. High-fat diet leads to adiposity and adipose tissue inflammation: the effect of whey protein supplementation and aerobic exercise training. Appl Physiol Nutr Metab. 2019;44(3):255-262; doi: 10.1139/apnm-2018-0307.

11. Maillard F, Vazeille E, Sauvanet P, Sirvent P, Combaret L, Sourdrille A, et al. High intensity interval training promotes total and visceral fat mass loss in obese Zucker rats without modulating gut microbiota. PLoS One. 2019;14(4):e0214660; doi: 10.1371/journal.pone. 0214660 .

12. Antunes LC, Elkfury JL, Jornada MN, Foletto KC, Bertoluci MC. Validation of HOMA-IR in a model of insulin-resistance induced by a high-fat diet in Wistar rats. Arch Endocrinol Metab. 2016;60(2):138-142; doi: 10.1590/2359-3997000000169.

13. Lebeck J. Metabolic impact of the glycerol channels AQP7 and AQP9 in adipose tissue and liver. J Mol Endocrinol. 2014;52(2):R165-R178; doi: 10.1530/JME13-0268.

14. Rotondo F, Ho-Palma AC, Remesar X, Fernández-López JA, del Mar Romero M, Alemany M. Glycerol is synthesized and secreted by adipocytes to dispose of excess glucose, via glycerogenesis and increased acyl-glycerol turnover. Sci Rep. 2017;7(1):8983; doi: 10.1038/ s41598-017-09450-4.

15. Catalán V, Gómez-Ambrosi J, Pastor C, Rotellar F, Silva C, Rodríguez A, et al. Influence of morbid obesity and insulin resistance on gene expression levels of AQP7 in visceral adipose tissue and AQP9 in liver. Obes Surg. 2008;18(6):695-701; doi: 10.1007/s11695-008-9453-7.

16. Kishida K, Kuriyama H, Funahashi T, Shimomura I, Kihara S, Ouchi N, et al. Aquaporin adipose, a putative 


\section{HUMAN MOVEMENT}

Y. Noorishorabi et al., Effects of HIIT on aquaglyceroporins

glycerol channel in adipocytes. J Biol Chem. 2000; 275(27):20896-20902; doi: 10.1074/jbc.M001119200.

17. Lee D-H, Park D-B, Lee Y-K, An C-S, Oh Y-S, Kang $\mathrm{J}-\mathrm{S}$, et al. The effects of thiazolidinedione treatment on the regulations of aquaglyceroporins and glycerol kinase in OLETF rats. Metabolism. 2005;54(10):12821289; doi: 10.1016/j.metabol.2005.04.015.

18. Prudente S, Flex E, Morini E, Turchi F, Capponi D, De Cosmo S, et al. A functional variant of the adipocyte glycerol channel aquaporin 7 gene is associated with obesity and related metabolic abnormalities. Diabetes. 2007;56(5):1468-1474; doi: 10.2337/db06-1389.

19. Ceperuelo-Mallafré V, Miranda M, Chacón MR, Vilarrasa N, Megia A, Gutiérrez C, et al. Adipose tissue expression of the glycerol channel aquaporin-7 gene is altered in severe obesity but not in type 2 diabetes. J Clin Endocrinol Metab. 2007;92(9):3640-3645; doi: 10.1210/jc.2007-0531.

20. Rodríguez A, Catalán V, Gómez-Ambrosi J, Frühbeck G. Role of aquaporin-7 in the pathophysiological control of fat accumulation in mice. FEBS Lett. 2006;580(20): 4771-4776; doi: 10.1016/j.febslet.2006.07.080.

21. Arner P. Human fat cell lipolysis: biochemistry, regulation and clinical role. Best Pract Res Clin Endocrinol Metab. 2005;19(4):471-482; doi: 10.1016/j.beem.2005. 07.004 .

22. Rodríguez A, Catalán V, Gómez-Ambrosi J, Frühbeck G. Visceral and subcutaneous adiposity: are both potential therapeutic targets for tackling the metabolic syndrome? Curr Pharm Des. 2007;13(21):2169-2175; doi: 10.2174/138161207781039599.

23. Gena P, Mastrodonato M, Portincasa P, Fanelli E, Mentino D, Rodríguez A, et al. Liver glycerol permeability and aquaporin-9 are dysregulated in a murine model of non-alcoholic fatty liver disease. PLoS One. 2013; 8(10):e78139; doi: 10.1371/journal.pone.0078139.

24. Gena P, Del Buono N, D’Abbicco M, Mastrodonato M, Berardi M, Svelto M, et al. Dynamical modeling of liver aquaporin-9 expression and glycerol permeability in hepatic glucose metabolism. Eur J Cell Biol. 2017; 96(1):61-69; doi: 10.1016/j.ejcb.2016.12.003.

25. Cai C, Wang C, Ji W, Liu B, Kang Y, Hu Z, et al. Knockdown of hepatic aquaglyceroporin-9 alleviates high fat diet-induced non-alcoholic fatty liver disease in rats. Int Immunopharmacol. 2013;15(3):550-556; doi: 10.1016/j.intimp.2013.01.020.

26. Rocha-Rodrigues S, Rodríguez A, Becerril S, Ramírez B, Gonçalves IO, Beleza J, et al. Physical exercise remodels visceral adipose tissue and mitochondrial lipid metabolism in rats fed a high-fat diet. Clin Exp Pharmacol Physiol. 2017;44(3):386-394; doi: 10.1111/1440-1681. 12706.

27. Londos C, Brasaemle DL, Schultz CJ, Adler-Wailes DC, Levin DM, Kimmel AR, et al. On the control of lipolysis in adipocytes. Ann N Y Acad Sci. 1999;892:155168; doi: 10.1111/j.1749-6632.1999.tb07794.x.
28. Wewege M, van den Berg R, Ward RE, Keech A. The effects of high-intensity interval training vs. moderate-intensity continuous training on body composition in overweight and obese adults: a systematic reviewandmeta-analysis.ObesRev.2017;18(6):635-646; doi: 10.1111/obr.12532.

29. Kawano Y, Cohen DE. Mechanisms of hepatic triglyceride accumulation in non-alcoholic fatty liver disease. J Gastroenterol. 2013;48(4):434-441; doi: 10.1007/ s00535-013-0758-5. 